\title{
Optimal Distribution Grid Operation Using Demand Response
}

\author{
Ricardo Faia, Bruno Canizes, Pedro Faria \\ GECAD, Polytechnic of Porto \\ Porto, Portugal \\ $\{$ rfmfa,bmrc,pnf $\} @$ isep.ipp.pt
}

\author{
Zita Vale \\ Polytechnic of Porto (ISEP/IPP) \\ Porto, Portugal \\ zav@isep.ipp.pt
}

\author{
José M. Terras, Luís V. Cunha \\ InovGrid European Agenda Office \\ EDP - Distribuição Energia, S.A. \\ Lisboa, Portugal \\ \{josemanuel.terras, luis.cunha\}@edp.pt
}

\begin{abstract}
This paper presents a model in which distribution system operator can take advantage of the flexibility of endusers to overcome some operation issues on low voltage networks. Distributed generation based on renewable energy sources connected to these kinds of networks are challenging the conventional control and operation framework designed for passive distribution networks. Considering this, the proposed research work presents an approach based on mixed integer quadratic problem using the flexibility available in the endusers to minimize the operation costs of distribution system operator. For this goal, it is seeking the minimization of power losses and flexibility acquisition costs. The presented case study considers a realistic low voltage network with 256 bus and 96 connected end-users to show the approach effectiveness.
\end{abstract}

Keywords-Distribution System Operator, End-users, Flexibility, Low Voltage Networks, Network Operation.

\section{INTRODUCTION}

Nowadays, many people move to cities in search of a better quality of life, and this contributes to the continuous expansion of urban areas, which play a significant role in modern economies. However, the urban population is responsible for most greenhouse gas emissions, and the United Nations estimates that the urban population will reach $70 \%$ of the world's total population by 2050 [1]. Consequently, it is necessary to make intelligent use of resources in urban environments.

The increasing electricity needs can be related to the consumer's new requirements, for instance, an increase in electrical based home equipment. Several innovative developments in power distribution systems have taken place around the world. One of them is related to the minimization of the carbon footprint using a large-scale integration of renewable energy sources (RES) such as wind and solar. The European Union (EU) renewable energy directive (2009/28/EC) [2] sets a binding target of $20 \%$ final energy consumption from renewable sources by 2020. In December 2018, the new revised renewable energy directive (2018/2001)[3] entered into force by establishing a new binding renewable energy target for the EU for 2030 of at least $32 \%$, with a clause for a possible upwards revision by 2023 .

A significant amount of distributed generation (DG) based on RES has been integrated and installed in the low voltage (LV) networks to answer to the governmental requirements. Due to this RES integration the actual LV network operation mode considers a bi-directional power flow instead of a

This work has received funding from the European Union's Horizon 2020 research and innovation programme under project DOMINOES (grant agreement No 771066), from FEDER Funds through COMPETE program and from National Funds through (FCT) under the project UID/EEA/00760/2019, and Ricardo Faia is supported by national funds through FCT with PhD grant reference SFRH/BD/133086/2017. unidirectional power flow (from main source to consumer). Thus, new technical challenges for the operation of the LV networks arise [4].

IEC 60038 standard (international recommendation), the voltage of three-phase and four wires networks is $230 / 400 \mathrm{~V}$ [5]. In power systems, the last stage of the network operates in LV level (230 / $400 \mathrm{~V})$, where the small-scale customers and suppliers use this voltage level. In this voltage level the customers and suppliers have a small capacity. However, the network in this level has a massive number of bus. The LV feeder construction has a lower cost when compared with medium and high voltage levels. Due to the simplicity protection schemes design for most of LV networks the radial network topology is used [6].

Different researchers around the world have proposed several planning and operational schemes for LV networks. In reference [7] a flywheel energy storage systems are used for improving the power quality of the network, and can also provide energy for a black start, unbalance compensation and can be a spinning reserve. The authors present a control mechanism based on the ancillary services required in the LV network.

A different approach is considered in [8], the authors present a method for voltage management in LV networks. They are considering the implementation of optimal residential (DR) and on-load tap changers. In reference [9], an optimization model for economic operation of micro-grid is considered. The presented model minimizes the micro-grid system operation costs making full use of the clean energy considering DG and DR. The use of DR action in end-users level was implemented in [10], and demonstrated the financial benefits that can be obtained from the correct use of the flexibility using equipment already installed in the household.

This paper proposes an optimization model that can be used as a decision-support tool to assist the DSO in the operation of the distribution LV network making use of the flexibility of the demand side and considering the bidirectional power flow. For this propose the demand side flexibility represents the part of the load that can be changed without violating the comfort standards of the users. The application of DR concept in this paper is performed to obtain flexibility in end-users side. The main goal of the optimization model is minimizing the DSO expenditures of the network operation. To this end, the proposed methodology seeks the following: Minimize power losses costs; and Minimize the costs considering the flexibility acquisition.

This paper is organized as follows: Section II presents the proposed methodology; Section III describes the case study, while the results are presented in Section IV. Finally, Section $\mathrm{V}$ presents the conclusions. 


\section{Proposed METHEdOLOGY}

The proposed methodology is shown with the presenting of the optimization problem formulation. The objective function minimizes the total operation cost (TOC) and can be formulated as (1).

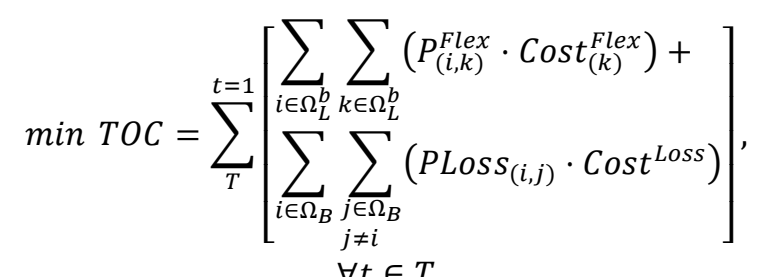

The objective function (1) is subject to the constraints (2)(16). The voltage magnitude of each bus is constrained by a maximum and minimum deviation (2). The maximum and minimum angle deviation is constrained by (3).

$$
\begin{aligned}
& V_{(i, t)}^{\min } \leq V_{(i, t)} \leq V_{(i, t)}^{\max } \quad \forall i \in \Omega_{B}, \forall t \in T \\
& \theta_{(i, t)}^{\min } \leq \theta_{(i, t)} \leq \theta_{(i, t)}^{\max } \quad \forall i \in \Omega_{B}, \forall t \in T
\end{aligned}
$$

Constraint (4) guarantees the active power balance in each distribution network bus.

$$
\begin{gathered}
\sum_{i \in \Omega_{D G}^{n d}} P_{D G(i, t)}+\sum_{i \in \Omega_{B S}^{b}} P_{\text {Supplier }(i, t)}- \\
\sum_{i \in \Omega_{L}^{b}} \sum_{k \in \Omega_{L}^{b}}\left(P_{\text {Load }(i, k, t)}-P_{(i, k, t)}^{\text {Flex }}\right)- \\
\sum_{i \in \Omega_{B}} P_{\text {Inj }(i, t)}=0, \forall t \in T
\end{gathered}
$$

Constraint (5) guarantees the reactive power balance in each distribution network bus.

$$
\begin{gathered}
\sum_{i \in \Omega_{B S}^{b}} Q_{\text {Supplier }(i, t)}-\sum_{i \in \Omega_{L}^{b}} Q_{\text {Load }(i, t)}- \\
\sum_{i \in \Omega_{B}} Q_{\text {Inj }(i, t)}=0, \forall t \in T
\end{gathered}
$$

This equation (6) represents the injected active power in each bus of the network.

$$
\begin{gathered}
P_{\text {Inj }(i, t)}=V_{(i, t)} \cdot \sum_{j \in \Omega_{B}} V_{(j, t)}\left(G_{(i, j, t)} \cdot \cos \theta_{(i, j, t)}\right. \\
\left.+B_{(i, j, t)} \cdot \sin \theta_{(i, j, t)}\right)
\end{gathered}
$$

$\forall i \in \Omega_{B}, \forall(i, j) \in \Omega_{l}, \quad \forall t \in T$

The injected reactive power in each bus is represented by equation (7).

$$
\begin{aligned}
& \sum_{\text {Inj }(i, t)}=V_{(i, t)} . \\
& \sum_{\left(j \in \Omega_{B}\right.}\left(G_{(i, j, t)} \cdot \sin \theta_{(i, j, t)}-B_{(i, j, t)} \cdot \cos \theta_{(i, j, t)}\right) \\
& \forall i \in \Omega_{B}, \forall(i, j) \in \Omega_{l}, \quad \forall t \in T
\end{aligned}
$$

The active power flow for each network line is given by equation (8).

$$
\begin{aligned}
& P_{(i, j, t)}=\left(V_{(i, t)}^{2}-V_{(i, t)} \cdot V_{(j, t)} \cdot \cos \theta_{(i, j, t)}\right) \cdot G_{(i, j, t)} \\
& -\left(V_{(i, t)} \cdot V_{(j, t)} \cdot \sin \theta_{(i, j, t)}\right) \cdot B_{(i, j, t)} \\
& \forall i \in \Omega_{B}, \forall j \in \Omega_{B}, \forall(i, j) \in \Omega_{l}, \forall t \in T
\end{aligned}
$$

Equation (9) gives the reactive power flow for each line.

$$
\begin{aligned}
& Q_{(i, j, t)}=-\left(V_{(i, t)}^{2}-V_{(i, t)} \cdot V_{(j, t)} \cdot \cos \theta_{(i, j, t)}\right) \cdot B_{(i, j, t)} \\
& -\left(V_{(i, t)} \cdot V_{(j, t)} \cdot \sin \theta_{(i, j, t)}\right) \cdot G_{(i, j, t)} \\
& \forall i \in \Omega_{B}, \forall j \in \Omega_{B}, \forall(i, j) \in \Omega_{l}, \forall t \in T
\end{aligned}
$$

The apparent power flow equation, in equation (10), is given by the square root of the active power flow and reactive power flow squares.

$$
\begin{gathered}
F \operatorname{Flow} S_{(i, j, t)}=\sqrt{P_{(i, j, t)^{2}+Q_{(i, j, t)}{ }^{2}},} \\
\forall(i, j) \in \Omega_{l}, \forall t \in T
\end{gathered}
$$

The active power loss of each line is represented by equation (11).

$$
\begin{aligned}
& \operatorname{PLoss}_{(i, j, t)}=\frac{P_{(i, j, t)}^{2}+Q_{(i, j, t)}^{2}}{V_{(i, t)}^{2}} \cdot r_{(i, j)} \\
& \forall i \in \Omega_{B}, \quad \forall(i, j) \in \Omega_{l}, \forall t \in T
\end{aligned}
$$

The maximum power flow in each line is given by (12).

$$
\begin{gathered}
0 \leq F \operatorname{low}_{(i, j, t)} \leq F \operatorname{Flow} S_{(i, j)}^{\max } \\
\forall(i, j) \in \Omega_{l}, \forall t \in T
\end{gathered}
$$

The active power is constrained by the maximum and minimum capacity that can be supplied (13).

$$
\begin{aligned}
& P_{\text {SMinLimit }(b s)} \leq P_{\text {Supplier }(b s, t)} \leq P_{\text {SMaxLimit }(b s)} \\
& \forall b s \in \Omega_{B S}, \forall t \in T
\end{aligned}
$$

The reactive power is constrained by the maximum and minimum capacity that can be supplied (14).

$$
\begin{aligned}
& Q_{\text {SMinLimit }(b s)} \leq Q_{\text {Supplier }(b s, t)} \leq Q_{S M a x L i m i t}(b s) \\
& \forall b s \in \Omega_{B S}, \forall t \in T
\end{aligned}
$$

Constraint (15) guarantees that load flexibility per client must be lower or equal to the maximum load flexibility per client.

$$
0 \leq P_{(i, k, t)}^{\text {Flex }} \leq P_{(i, k, t)}^{\text {FlexMax }} \quad \forall k \in \Omega_{L}^{b}, \forall t \in T
$$

The summation of load flexibility in the system must be lower or equal to the maximum load flexibility (16).

$$
\sum_{i \in \Omega_{L}^{b}} \sum_{k \in \Omega_{L}^{b}} P_{(i, k, t)}^{\text {Flex }} \leq P_{t}^{\text {FlexTotal }}, \forall t \in T
$$

\section{CASE STUdy}

The presented case study is a part of a realistic LV distribution network. This network has 236 buses, 235 lines, 96 connected end-users and is supplied by a MV connection with a transformer (Figure 1).-End users have contracts with the system operator to reduce their consumption when is required. All end-users have installed in your household equipment with ensuring perform the cut of the required request.

The end-users are divided into three different groups: the group 1 has a contracted power of $1.15 \mathrm{kVA}$ and a simple tariff. This group has 26 end-users. Group 2 has 32 end-users, with a $2.3 \mathrm{kVA}$ of contracted power and a simple tariff. The group 3 consists in 38 end-users with a bi-hour tariff and a contracted power of $3.45 \mathrm{kVA}$. The user's classification was performed considering the maximum consumption of each one of them. It is worth to refer that the DR tariff is created based on the electricity tariff of each group. This idea is based on [11], where was presented a similar approach for determining the DR tariff. Figure 2 presents the prices for DR reduction considering the 24 hours of the day. As can be seen 
in this figure the DR tariff the tariff for group 3 (bi-hour tariff) is variable according to the time period.

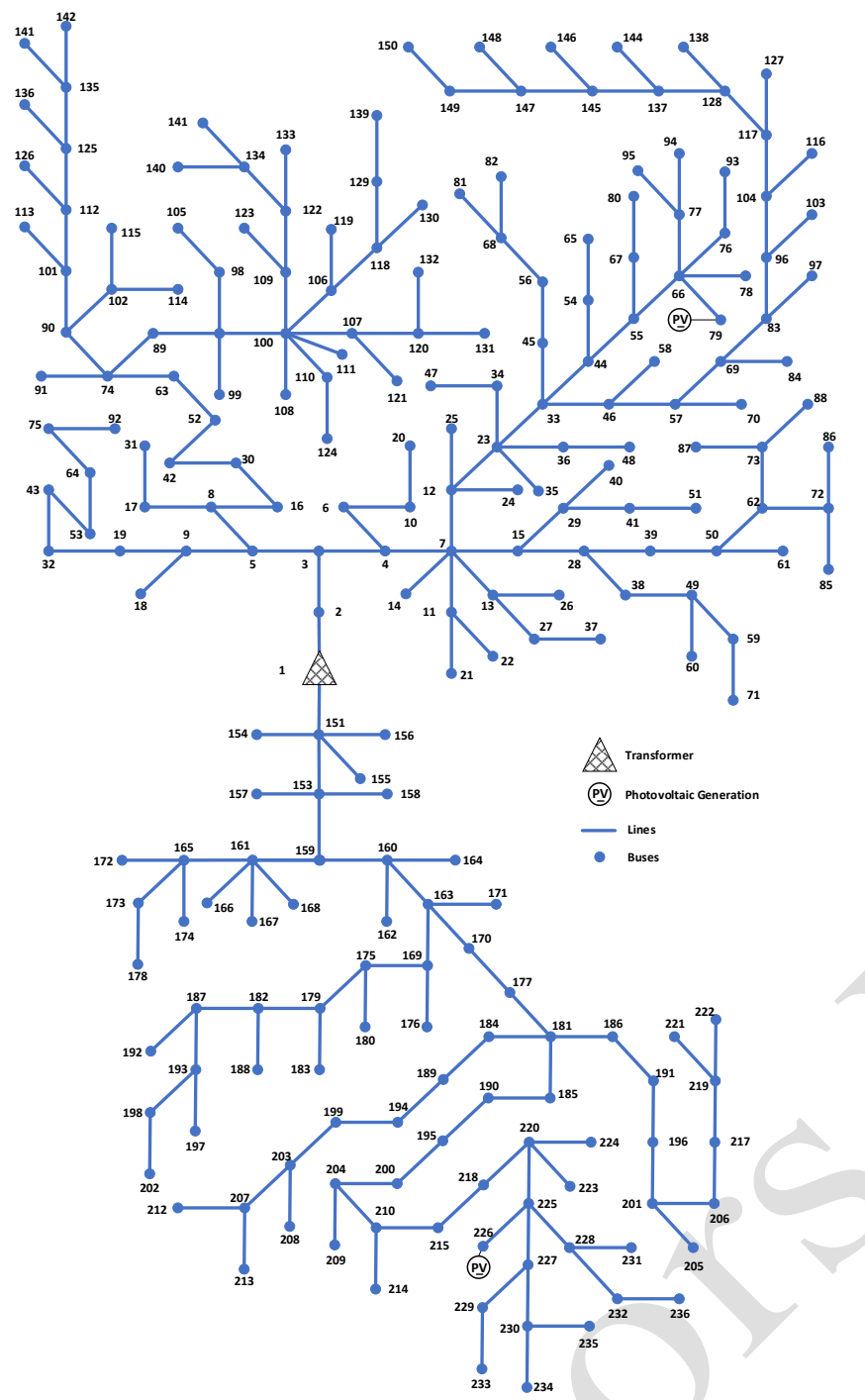

Figure 1. Computational network design (Disclaimer: The representation above does not reflect the real configuration of the network)

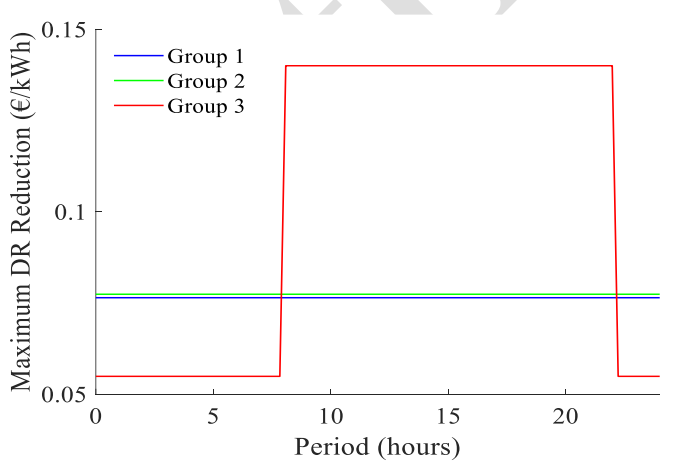

Figure 2. DR Tariff

TABLE I depicts the electricity and DR tariffs for each group. The peak and off-peak represent different periods of the day. The peak periods include the hours between 8:00 to 22:00 and the off-peak period includes the 1:00 to 8:00 and 22:00 to 24:00 hours. In group 1 and group 2 the DR tariff is $0.5 \times$ Eletricity price. Group 3 has two different prices according to the period of the day. In peak hours the DR tariff is $0.75 \times$ Eletricity price and in off-peak is $0.5 \times$
Eletricity price. Figure 3 presents the input data for the total network consumption and maximum DR reduction.

TABLE I. ELETRICITY PRICES AND DR PRICES

\begin{tabular}{|l|l|l|l|l|}
\hline & \multicolumn{3}{|l|}{ Electricity price $(€ / \mathrm{kWh})$} & \multicolumn{2}{l|}{ DR price $(€ / \mathrm{kWh})$} \\
\hline & Peak & Off-peak & Peak & Off-peak \\
\hline Group 1 & \multicolumn{2}{|c|}{0.153} & \multicolumn{2}{|c|}{0.076} \\
\hline Group 2 & \multicolumn{2}{|c|}{0.155} & \multicolumn{2}{c|}{0.077} \\
\hline Group 3 & 0.187 & 0.110 & 0.140 & 0.055 \\
\hline
\end{tabular}

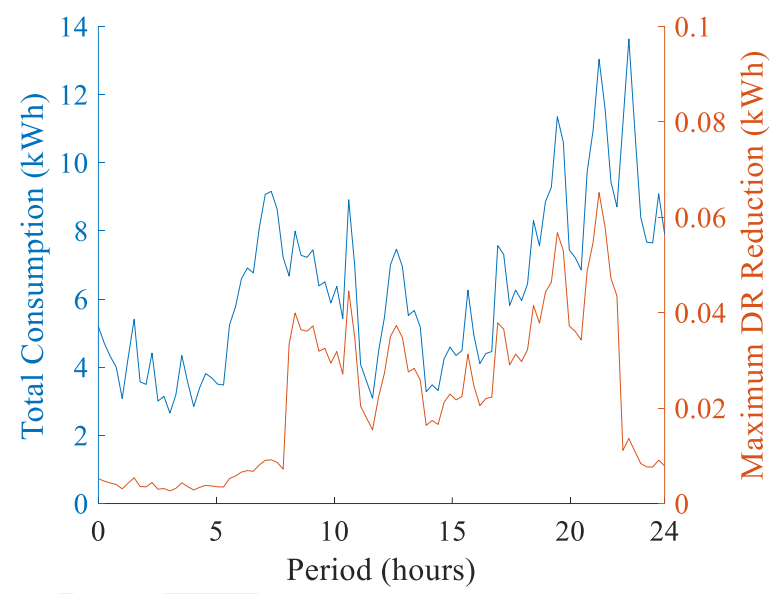

Figure 3. Total Consumption and maximum DR reduction

Figure 3 has two different y-axes with two different scales, but both in $\mathrm{kWh}$. Analysing this figure, the consumption peak is verified during the night hours (21:00 and 23:00). When the period belongs to the peak hours the maximum DR value in each period is $0.001 \times$ total network consumption, if considered a off-peak period the maximum DR reduction is $0.005 \times$ total network consumption. Figure 4 presents the mean consumption for each group of consumers.

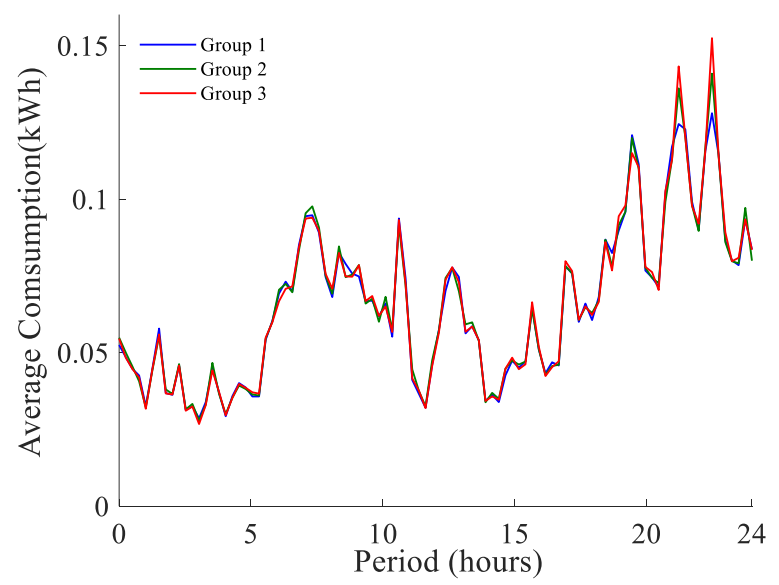

Figure 4. Mean Consumption

The mean profile is very similar for each, as can be seen in Figure 4. Group 3 present the highest mean consumption between groups. Analysing this figure, the period 21:00 and 23:00 presents the highest values. Figure 5 presents the maximum value reduction for each end-user group. The reduction values were determined based on the previous study from reference [10]. Through Figure 5 group 3 presents the maximum possible contribution for DR application. The maximum DR reduction is obtained considering the minimum value of energy consumption for each group. For group 1 the 
maximum DR reduction is $0.0008 \times$ minimun consumption group 1 , in the case of group 2 is $0.0015 \times$ minimum consumption group 2 and for group 3 have two different values considering the two different periods: peak $0.015 \times$ minimum consumption group 3 and off-peak $0.002 \times$ minimum consumption group 3 .

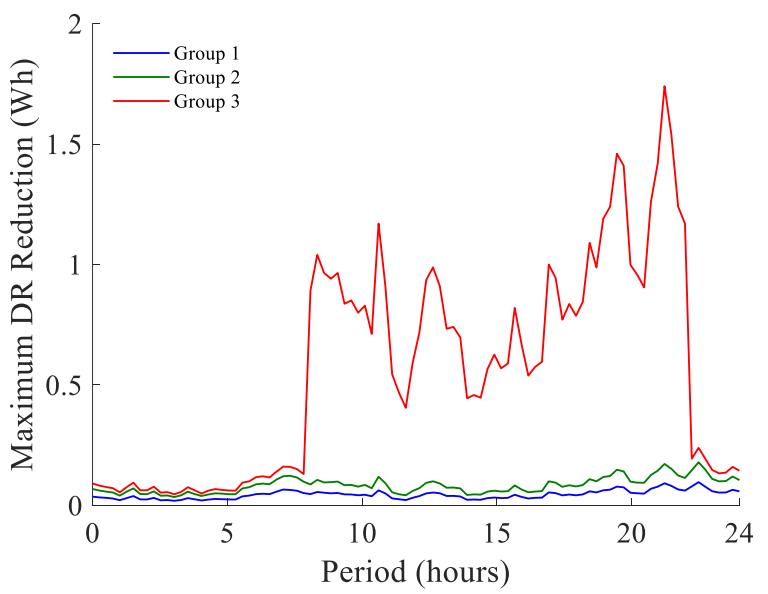

Figure 5. Maximum DR per group

The case study can be summarized as follows:

- $\quad$ Three different tariffs for DR, based on the electricity tariff of each end-user (three different groups);

- The total maximum DR reduction is limited in each period;

- Each end-user has a different maximum value of DR reduction;

- Each group of end-users has a total maximum value of DR reduction;

- The losses cost is considered linear, $0.12 € / \mathrm{kW}$.

The proposed work was developed in MATLAB R2018a, and TOMLAB 8.164 bits using a computer with one Intel Xeon E5-2620 v2 processor and 16 GB of RAM running Windows 10 Pro.

\section{RESUlTS AND DisCUSSION}

This section presents the results for one day operation with 96 periods (00:15 hours each). The actual scenario, name as baseline (without DR) is used as reference case to compare with the scenario when DR reduction possibility is considered. TABLE II presents a comparison of the results. The results are presented for two different scenarios the baseline and the use of DR.

TABLE II. RESULTS COMPARISON

\begin{tabular}{|l|l|l|l|}
\hline Scenario & $\begin{array}{l}\text { Total Operation } \\
\text { Costs }(€)\end{array}$ & $\begin{array}{l}\text { Losses } \\
\text { Costs }(€)\end{array}$ & $\begin{array}{l}\text { DR } \\
\text { costs }(€)\end{array}$ \\
\hline Baseline & 0.27 & 0.27 & 0.00 \\
\hline With DR & 0.26 & 0.23 & 0.03 \\
\hline
\end{tabular}

By TABLE II it is possible to see that the baseline scenario presents the higher total operation costs value compared with the total operation cost vale considering DR. The difference is $0.01 €$ and represents a reduction of $3 \%$.

The cost of power losses in the scenario with DR is smaller than in the baseline scenario. The difference is $0.04 €$ and it is related to the consumption reduction of the end-users. The DR cost is $0.03 €$ and represent approximately $10 \%$ of the total operation costs. On the other hand, the losses cost in the scenario with DR represents $90 \%$ of the total operation costs. TABLE III and Figure 7 presents the results of the DR reduction.

The operation total cost results for 24 hours are presented in Figure 6. Each hour is divided into 4 different periods, each period has 15 minutes of duration. Through Figure 6 it can be seen two different values of operations costs. In blue it is presented the results of baseline scenario and in red the results for the scenario with DR.

With Figure 6 it is possible to see that there are periods when the operation costs have different values. These periods are presented in TABLE III. The DR reduction is used in 13 periods, in which 5 are in off-peak periods and 8 in peak periods. As can be seen in TABLE III the total DR reduction is $0.231 \mathrm{~kW}$, in which 0.077 are in off-peak periods and 0.213 are in peak periods. The period 84 at 20:45 is the period with higher DR reduction that corresponds the period with the most expensive DR costs. For DSO the costs of DR reduction have an average cost of $0.107 € / \mathrm{kW}$ and the DR average reduction in peak periods is $0.018 \mathrm{~kW}$ and in off-peak is $0.009 \mathrm{~kW}$.

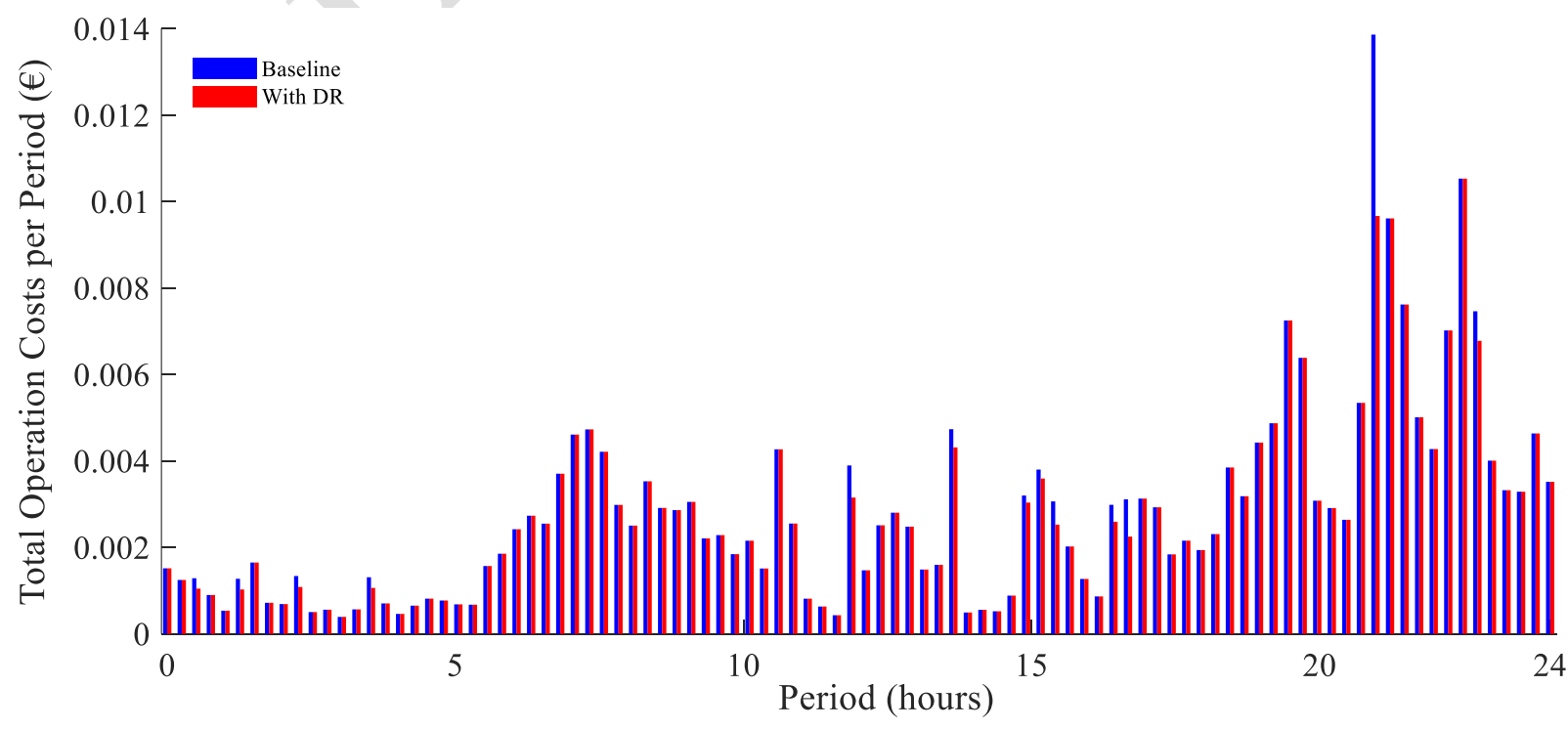

Figure 6. Total operation costs $€$ 
TABLE III. DR REDUCTION PERIODS

\begin{tabular}{|l|l|l|l|}
\hline Hours & Period & DR Cost $(€)$ & DR reduction $(\mathrm{kWh})$ \\
\hline $0: 30$ & 3 & 0.0002 & 0.004 \\
\hline $1: 15$ & 6 & 0.0002 & 0.004 \\
\hline $2: 15$ & 10 & 0.0003 & 0.004 \\
\hline $3: 30$ & 15 & 0.0002 & 0.004 \\
\hline $11: 45$ & 48 & 0.0030 & 0.022 \\
\hline $13: 30$ & 55 & 0.0034 & 0.026 \\
\hline $14: 45$ & 60 & 0.0022 & 0.016 \\
\hline $15: 00$ & 61 & 0.0029 & 0.022 \\
\hline $15: 15$ & 62 & 0.0020 & 0.015 \\
\hline $16: 15$ & 66 & 0.0020 & 0.015 \\
\hline $16: 30$ & 67 & 0.0021 & 0.016 \\
\hline $20: 45$ & 84 & 0.0073 & 0.055 \\
\hline $22: 30$ & 91 & 0.0007 & 0.011 \\
\hline
\end{tabular}

Analysing the Figure 7 it is possible to have a better perspective of when the reduction is performed and in what are the reduced amount.

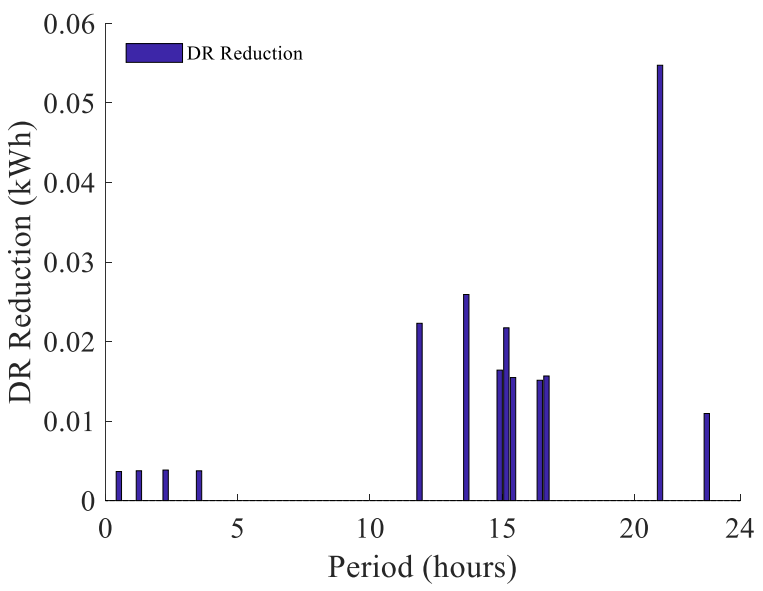

Figure 7. DR reduction in each period

TABLE IV presents the results of total DR reduction per group of consumers. As we are in presence of different tariffs it was expected that the amount of energy reduction by each group is different.

TABLE IV. DR REDUCTION FOR EACH GROUP

\begin{tabular}{|l|l|l|l|}
\hline & Group 1 & Group 2 & Group 3 \\
\hline Total DR (kWh) & 0.009 & 0.025 & 0.179 \\
\hline Reduction number & 216 & 357 & 356 \\
\hline
\end{tabular}

The results in TABLE IV shows that group 3 has a higher value of total DR reduction. The reduction amount in TABLE IV represents the number of times the DR reduction was performed over the 96 periods, i.e., the end-users of group 3 performed $356 \mathrm{DR}$ reduction action in all periods. With TABLE IV is possible to conclude that group 3 is most receptive to receive DR action. TABLE V presents the results of DR reduction, and total demand in both scenarios. The feeder 1 includes the busses 2 to 150, and feeder 2 the busses 151 to 236.

TABLE V. DR REDUCTION FOR EACH FEEDER

\begin{tabular}{|l|l|l|}
\hline & Feeder 1 & Feeder 2 \\
\hline Total DR (kWh) & 0.155 & 0.059 \\
\hline Total demand (with DR) $(\mathrm{kWh})$ & 383.389 & 218.640 \\
\hline Total demand (baseline) $(\mathrm{kWh})$ & 383.545 & 218.699 \\
\hline
\end{tabular}

As can be seen in TABLE $\mathrm{V}$ the feeder 1 has a higher demand in the baseline scenario and have the higher total DR reduction. The DR reduction of feeder 1 is $72 \%$ of the total DR reduction performed in the same duration (96 periods).

\section{CONCLUSION}

It was verified that the use of DR reduction possibility is advantageous in terms of total operational costs. The reduction in total operational cost is minimal, but the benefits may be more evident when considering a scenario with higher values of demand. The costs of power losses as shown by the results decreased in the scenario with DR. If the assumed prices of the DR tariff change, operating costs must change. There is a direct relationship between the proposed model and the DR tariff used. For future work, we intend to perform more experiments considering a higher number of distributed generators and a higher value for demand profiles. Different costs in objective function can be included (e.g., lines congestion cost). Additionally, we intend to perform an integrative analysis between medium voltage and LV to assess the impact of DR models in a wider grid perspective, to evaluate possible gains of interconnected mechanisms between different voltage levels and to gain operational scale.

\section{ACKNOWLEDGMENT}

This paper is the result of an integrative and collaborative partnership between the Polytechnic of Porto and EDP Distribuição - Energia S.A. developed within the Horizon 2020 Project DOMINOES (grant agreement No771066). The consumers' data used in this study are $100 \%$ anonymized, being all of the content associated with confidential and sensitive content compliant with the Regulation (EU) 2016/679 - General Data Protection Regulation - and other laws and regulations applicable to the electricity sector. Nevertheless, the anonymized data content associated to this study is subject of elimination until the end of the DOMI NOES project, on 31st of March of 2021.

A. Paaso, D. Kushner, S. Bahramirad, and A. Khodaei, "Grid Modernization Is Paving the Way for Building Smarter Cities," IEEE Electrif. Mag., vol. 6, no. 2, 2018

[2] European Union, "Directive 2009/28/ec of the european parliament and of the council of 23 april 2009 on the promotion of the use of energy from renewable sources and amending and subsequently repealing directives. Off. J. Eur. Union, 2009 European Union, "Directive 2018/2001 on the promotion of the use of energy from renewable sources," Off. J. Eur. Union, 2018. R. Viral and D. K. Khatod, "Optimal planning of distributed generation systems in distribution system: A review," Renew. Sustain. Energy Rev, 2012.

[5] Schneider Electric, "Electrical Installation Guide," 2018

[6] J. Driesen and F. Katiraei, "Design for distributed energy resources," IEEE Power Energy Mag.,pp. 30-39, 2008.

[7] S. Karrari, M. Noe, and J. Geisbuesch, "High-speed Flywheel Energy Storage System (FESS) for Voltage and Frequency Support in Low Voltage Distribution Networks," 2018 IEEE 3rd IEPS 2018 - 2018.

[8] M. M. Rahman, A. Arefi, G. M. Shafiullah, and S. Hettiwatte, "A new approach to voltage management in unbalanced low voltage networks using demand response and OLTC considering consumer preference," Int. J. Electr. Power Energy Syst., 2018

[9] Y. Wang et al., "Energy management of smart micro-grid with response loads and distributed generation considering demand response," J. Clean. Prod., vol. 197, pp. 1069-1083, 2018.

[10] R. Faia, P. Faria, Z. Vale, and J. Spinola, "Demand Response Optimization Using Particle Swarm Algorithm Considering Optimum Battery Energy Storage Schedule in a Residential House," Energies . 2019.

[11] J. Spínola, P. Faria, and Z. Vale, "Photovoltaic inverter scheduler with the support of storage unit to minimize electricity bill," in Advances in Intelligent Systems and Computing, 2017. 